\title{
GENETICS
}

\section{X-linked acrogigantism-genetic characterization of a newly described paediatric growth disorder}

A previously uncharacterized form of early-onset paediatric gigantism, termed X-linked acrogigantism (X-LAG), is caused almost exclusively by microduplications on chromosome Xq26.3, reveal new findings published in the New England Journal of Medicine.

Gigantism, which is caused by excessive production and secretion of growth hormone, is an extremely rare and poorly understood disorder. Previous studies have identified

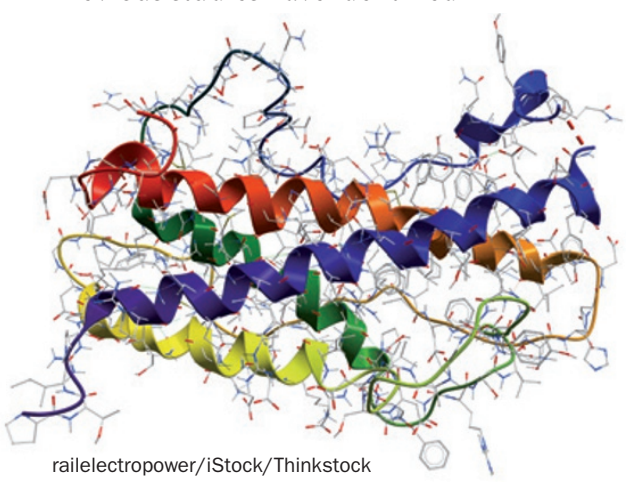

mutations in the MEN1 and AIP genes in adolescents and adults with acromegaly; however, the mechanisms that underlie early-onset paediatric gigantism have remained elusive.

A research group from the NIH, USA, headed by senior investigator Constantine Stratakis, performed genetic analysis on samples from 43 patients with gigantism who also had hypersecretion of growth hormone. Two phenotypes were identified among the patients on the basis of age of onset. One phenotype was characterized by rapid growth in early infancy and the other phenotype had typical onset in adolescence. Microduplications in Xq26.3 were found in samples from 13 patients, four of whom were from two unrelated families. The boundaries of the duplicated regions were unique to each patient, which suggested that the mutations arose through a replication-based mechanism. Additionally, all patients who carried these mutations developed features of gigantism before 5 years of age and, therefore, were considered to have X-LAG.

Strikingly, of the four genes located on the Xq26.3 region, only expression of GPR101 was found to be considerably deregulated in pituitary glands of patients with X-LAG. Overexpression of mutant GPR101 in a rat cell line led to increased secretion of growth hormone, which supports the hypothesis that GPR101 overexpression that results from microduplications in Xq26.3 causes X-LAG.

"This is pretty amazing," says Stratakis, "if there is no family history and you are dealing with a toddler with gigantism, based on these data, there is a more than $80 \%$ chance of having an Xq26 defect."

Jennifer Sargent

Original article Trivellin, G. et al. Gigantism and acromegaly due to Xq26 microduplications and GPR101 mutation. N. Engl. J. Med. doi:10.1056/NEJMoa1408028 


\section{CORRECTION}

X-linked acrogigantism-genetic characterization of a newly described paediatric growth disorder

Jennifer Sargent

Nat. Rev. Endocrinol. advance online publication 23 December 2014; doi:10.1038/nrendo.2014.230

In the online version of this article the name of the senior investigator was misspelled.

Strakatis should be Stratakis. This error has been corrected for the print and online versions of this article. 